\title{
Myoepithelial hamartoma of the distal ileum - A rare cause of adult intussusception
}

\author{
P JAGMOHAN, R ANAND, MK NARULA, V SINGHAL, RS SOLANKI
}

Ind J Radiol Imag 2006 16:2:185-188

Keywords: intussusception, adult, myoepithelial hamartoma, US, CT, BMFT

Introduction

Intussusception is defined as telescoping of a segment of the gastrointestinal tract into an adjacent one. It is the leading cause of intestinal obstruction and is one of the commonest causes of abdominal emergency in children (1). Adult intussusception however is rare with an incidence of 2-3 cases per million per year. An identifiable lead point that often requires definitive treatment is found in $70-90 \%$ cases. However unlike its childhood counterpart, adult intussusception has a subacute or chronic presentation and the diagnosis is often missed on clinical examination (2). The preoperative diagnosis can usually be made reliably by non-invasive imaging techniques like barium studies, US and CT.

Small bowel intussusceptions in most cases are secondary to benign lesions. We describe a case of leoileal intussusception in a middle aged male secondary to a myoepithelial hamartoma in the distal ileum which was detected on transabdominal US.

\section{Case Report}

A 53 year old man presented with complaints of intermittent pain in the epigastric and periumbilical region with significant weight loss for two years. Over the last year the patient had a few episodes of colicky pain with radiation of pain to the back and increase in intensity of pain. The physical examination was unremarkable. In view of the history of alcohol consumption and clinical presentation patient was referred for an abdominal US with the clinical diagnosis of chronic pancreatitis . Ultrasound abdomen revealed normal pancreas. In view of the repeated intermittent abdominal pain BMFT was done which showed a well defined filling defect in the distal ileal loops. A narrow column of barium was seen proximal to the defect. Distal jejunal loops were mildly dilated with delayed passage of contrast through small bowel loops (Fig 1). On US enteric intussusception with characteristic doughnut appearance was seen with alternating hypoechoic and hyperechoic layers. A well defined rounded $(2.42 .3 \mathrm{~cm})$ polypoid hypoechoic mass with smooth margins was seen at the distal end of intussusceptum. Few vessels were seen in the mass on colour flow imaging with convergence of vessels to the proximal central part suggesting a stalk (Fig 2,3). The diagnosis of enteric intussusception due to pedunculated polyp was made.

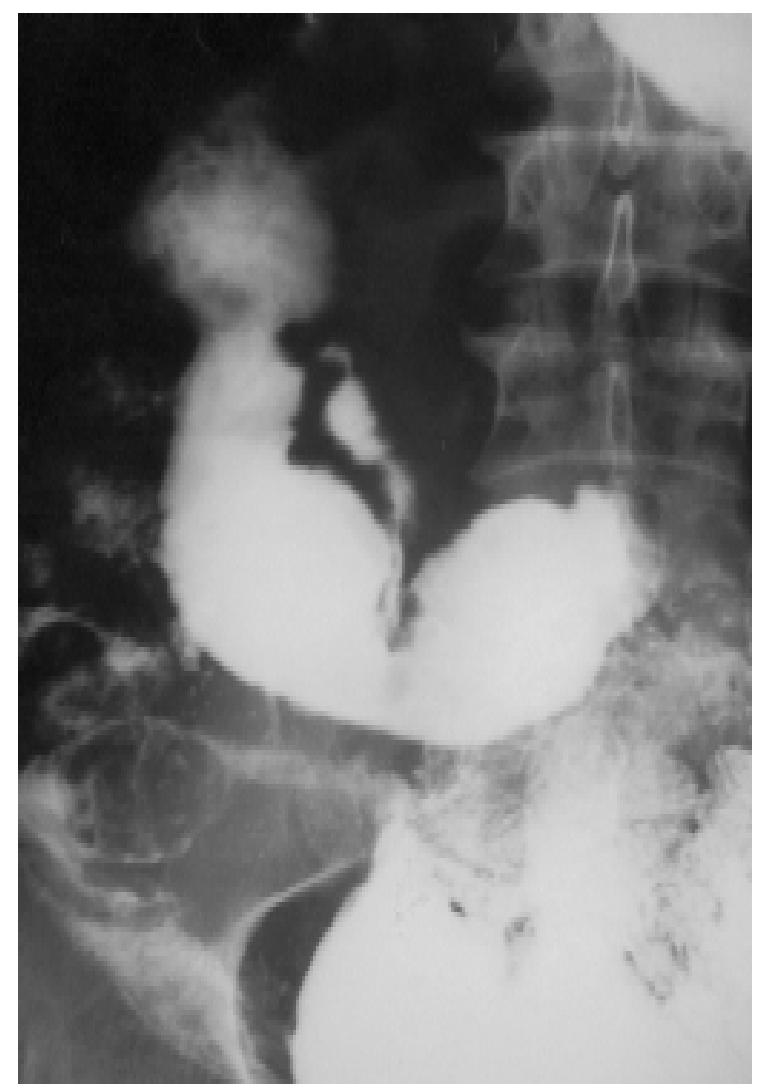

Fig.1. BMFT showing a well defined filling defect in the distal ileum with proximal narrowing and dilated jejunal loop.

From the Department of Radiodiagnosis, Lady Hardinge Medical College \& Department of Neurology, Jaipur Golden Hospital, New Delhi

Request for Reprints: Dr. Rama Anand, A-203, Meera Bagh, Outer Ring Road, New Delhi-110087

Received 20 October 2005; Accepted 15 April 2006 
$186 P$ Jagmohan et al
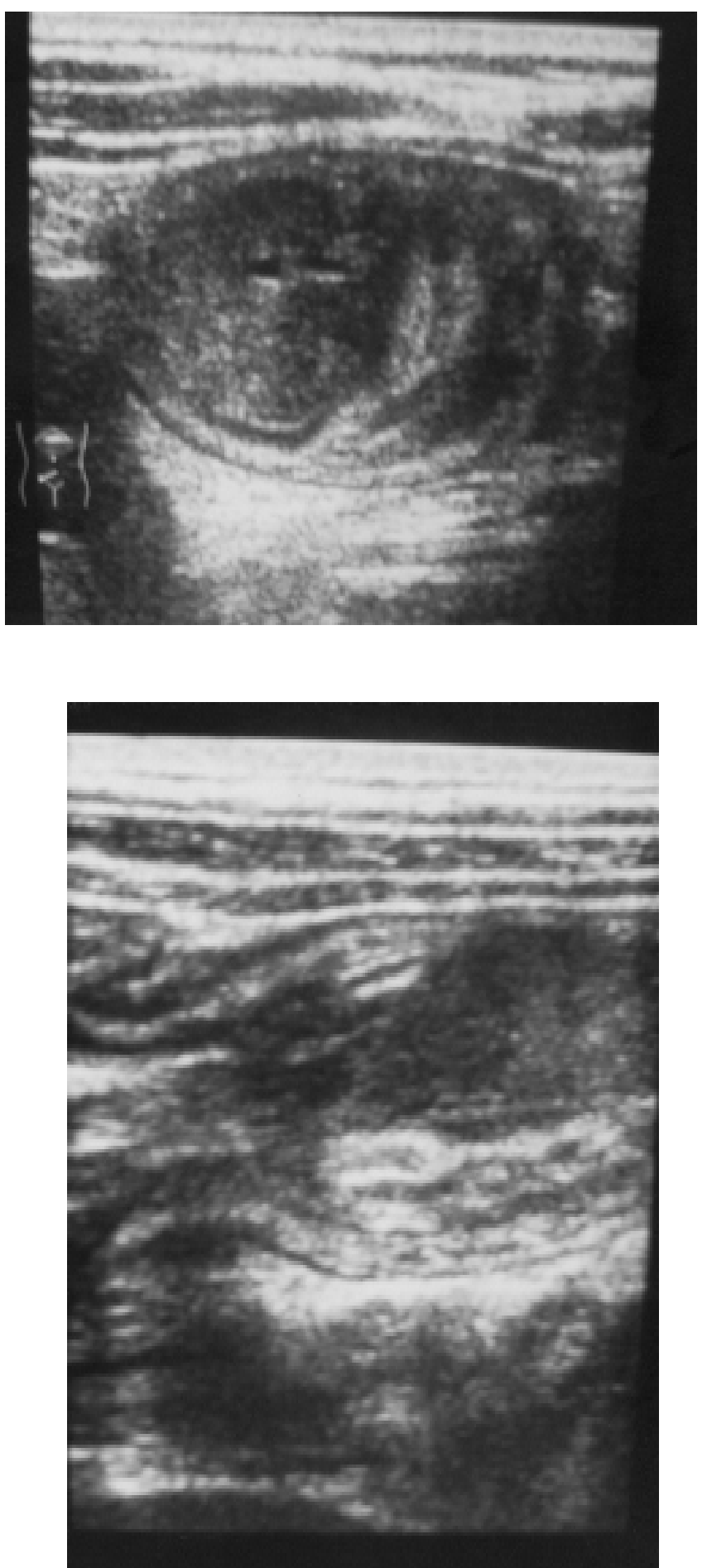

Fig.2a, b. Transabdominal US showing enteric intussusception with characteristic doughnut appearance on transverse scan (Fig.2a); multiple alternating hyperechoic and hypoechoic layers on longitudinal scan (Fig.2b). A well defined hypoechoic mass (Fig.2a,b) is seen eccentrically at the distal end of intussusceptum.

Computed tomography of the abdomen confirmed US finding of enteroenteric intussusception. Focal nodular thickening was seen along the posterior wall of bowel in the longitudinal scan, however the polyp seen on US could not be delineated on CT (Fig 4).
IJRI, 16:2, May 2006

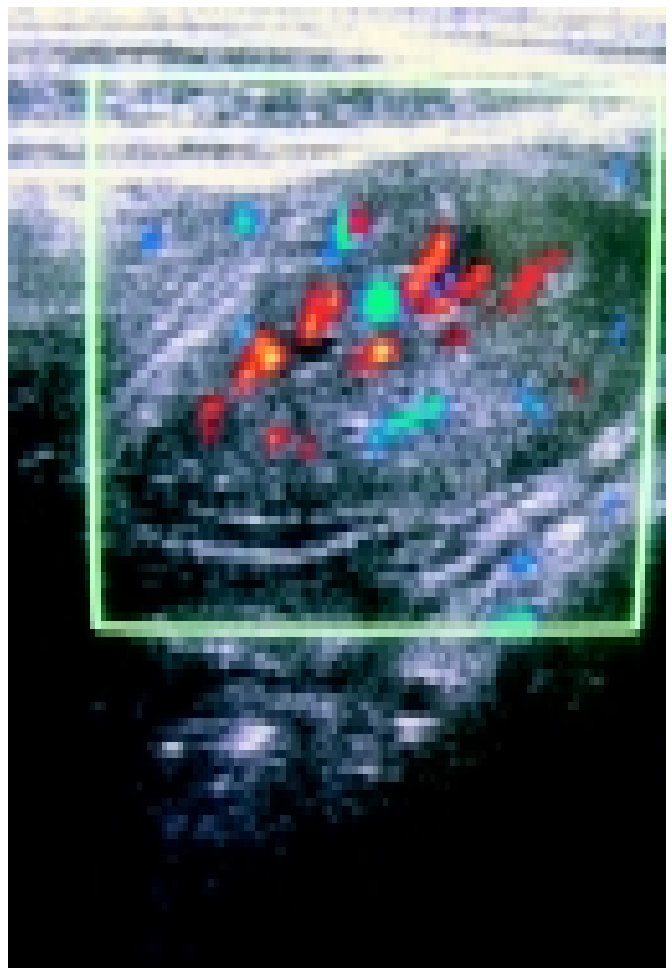

Fig.3. Color flow imaging shows few vessels within the mass with proximal convergence.
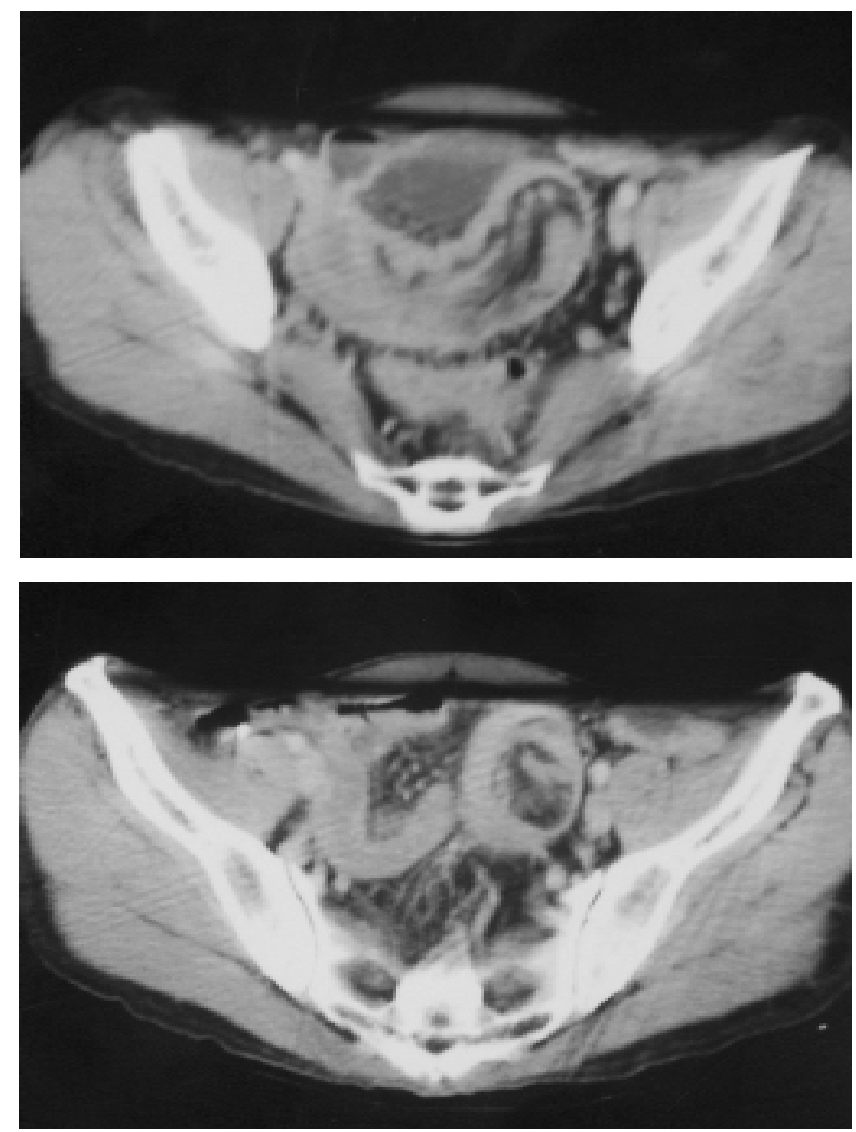

Fig.4a, b. CECT of enteroenteric intussusception: Longitudinal scan shows a sausage mass with focal nodular thickening posteriorly (Fig.4a); a target appearance is seen on transverse scan (Fig.4b) with eccentric mesenteric vessels and fat. 
Intraoperative findings confirmed radiographic findings. Ileoileal intussusception was noted with a $22 \mathrm{~cm}$ intraluminal pedunculated polyp acting as the lead point 4 feet from the ileocecal junction. Intussusception was reduced; ileal resection and anastomoses was done. Histopathological examination of the resected mass suggested a myoepithelial hamartoma. The postoperative period was unremarkable and there was no recurrence of symptoms on follow up.

\section{Discussion}

Childhood intussusception is idiopathic in $90 \%$ of cases. In contrast, adult intussusception has a demonstrable cause in $70-90 \%$ of cases including neoplasms, postoperative changes and adhesions, inflammation and miscellaneous conditions like Meckel's diverticulum and celiac disease $(1,3)$.

Benign lesions are responsible for approximately $25 \%$ of all adult intussusceptions with Peutz-Jeghers polyps and lipomas being common causes of small bowel intussusception (3). Malignant lesions of the small bowel like adenocarcinoma; malignant stromal tumors and metastases, most commonly from malignant melanoma are less common than benign neoplasms and are responsible for approximately $15 \%$ cases of small bowel intussusception (4). Myoepithelial hamartoma, also referred to as " ectopic pancreas" is a rare cause of enteric intussusception in adults. It is a developmental abnormality of displaced pancreatic tissue, smooth muscle and epithelial structures occurring in various amounts. If typical pancreatic acinar tissue is present, the lesion is referred to as "ectopic pancreas". When there is an abundance of smooth muscle and ductal structures, the term "myoepithelial hamartoma" is preferred. The hamartoma is usually solitary and under 3 have been reported in the more distal small bowel (5). Radiologically, a smooth surfaced non pedunculated lesion is seen with umbilication noted occasionally.

The most common symptoms of intussusception are intermittent abdominal pain, nausea and vomiting. Less frequent symptoms are malena, weight loss, fever and constipation. The preoperative diagnosis of adult intussusception is infrequent because of its vague clinical presentation and relative infrequency. However the increasing use of US and CT has improved the diagnostic ability (3). Tan et al reported a preoperative diagnostic rate of $80 \%$ in cases of intussusception using CT scan (2).

The classical features of intussusception on plain film like air crescent sign and presence of a soft tissue mass with decreased colonic air are neither sensitive nor specific, however plain radiograph may be useful in assessing signs of bowel compromise like pneumatosis and pneumoperitoneum (3).
The characteristic finding of intussusception on barium studies is the "coiled spring" appearance with a thin central barium stream with or without a leading mass. A " stretched spring" appearance in which the concentric barium rings are forced apart by increasing oedema in the bowel wall and mucosal folds has been suggested as a sign of vascular compromise (3).

The classic appearance of intussusception on transverse US scan is that of a target or doughnut mass with a hypoechoic rim representing the oedematous wall of the intussuscipiens and a central area of increased echogenicity representing intussusceptum and its invaginated mesenteric fat. A layering appearance with alternating hypoechoic and hyperechoic layers representing layers of bowel wall and mesentery is seen on longitudinal scan and a pseudokidney appearance is seen when the intussusception is visualized obliquely (3). Colour Doppler may be useful in determining the degree of vascular compromise of the involved bowel segments (3) . The characteristic doughnut appearance was seen in the present case on transverse scan with a multilayered appearance on longitudinal scan. In addition the leading mass was well demonstrated by US.

On CT a complex small bowel mass was seen with fat and mesenteric vessels seen between the opposing walls of intussusceptum and intussuscipiens similar to the observations made by previous authors $(3,6-8)$. However the leading mass which was well seen on US could not be demonstrated on CT. Abdominal CT has been shown to be the most accurate modality for indentification of intussusception $(3,6,7)$, however the underlying aetiology may be difficult to determine(3).

Magnetic resonance imaging can distinguish between liquid or solid components and ischemic or necrotic bowel can be detected. Motion insensitive sequences like T2 weighted HASTE demonstrated intussusception clearly, giving high contrast between intraluminal fluid and bowel wall $(3,6)$.

To conclude, intussusception in adults occurs rarely and differs from its paediatric counterpart in terms of aetiology, clinical presentation, diagnosis and treatment. Symptoms are usually non specific and of long duration and the condition is rarely suspected on clinical examination . A correct and timely diagnosis is necessary to prevent the complications of bowel infarction and perforation and to resect the underlying leading mass. Imaging thus plays a crucial role in the diagnosis and management of these patients.

\section{References :}

1. Agha FP. Intussusception in adults. AJR 1986; 146: $527-$ 531.

2. Tan KY, Tan S, Tan AGS, Chen CYY, Chng H, Hoe MNY. 
Adult intussusception: experience in Singapore. ANZ J Surg 2003; 73: 1044-1047.

3. Huang BY, Warshauer DM. Adult intussusception: diagnosis and clinical relevance. RCNA 2003; 41: 11371151.

4. Choi SH, Han JK, Kim SH etal. Intussusception in adults: from stomach to rectum. AJR 2004; 183: 691-698.

5. Olmsted WW, Ros PR, Hjermstad BM, McCarthy MJ, Dachman $\mathrm{AH}$. Tumors of the small intestine with little or no malignant predisposition: a review of the literature and report of 56 cases. Gastrointest Radiol !987; 12:
231-239.

6. Dawes LC, Hunt R, Wong JK, Begg S. Multiplanar reconstruction in adult intussusception: case report and literature review. Australasian Radiol 2004; 48: 74-76.

7. Steinwald PM, Trachiotis GD, Tannebaum IR. Intussusception in an adult secondary to an inverted Meckel's diverticulum. Anerican Surgeon 1996; 62: 889894.

8. Azar T, Berger DL. Adult intussusception. Ann Surg 1997; 226: 134-138. 